\title{
Upper Bounds of AZI and ABC Index for Transformed Families of Graphs
}

\author{
Muhammad Hussain, ${ }^{1}$ Muhammad Asif, ${ }^{1}$ Ashit Kumar Dutta, ${ }^{2}$ and Sultan Almotairi $\mathbb{D}^{3}$ \\ ${ }^{1}$ Department of Mathematics, COMSATS University Islamabad, Lahore 54000, Pakistan \\ ${ }^{2}$ Department of Computer Science and Information Systems, College of Applied Sciences, AlMaarefa University, \\ Riyadh 13713, Saudi Arabia \\ ${ }^{3}$ Department of Natural and Applied Sciences, Faculty of Community College, Majmaah University, \\ Majmaah 11952, Saudi Arabia
}

Correspondence should be addressed to Sultan Almotairi; almotairi@mu.edu.sa

Received 5 November 2021; Revised 10 December 2021; Accepted 29 December 2021; Published 25 January 2022

Academic Editor: Abdul Qadeer Khan

Copyright (c) 2022 Muhammad Hussain et al. This is an open access article distributed under the Creative Commons Attribution License, which permits unrestricted use, distribution, and reproduction in any medium, provided the original work is properly cited.

\begin{abstract}
Topological index is a mapping which corresponds underlying graph with a numeric value and invariant up to all the isomorphisms of graph. Our study is based on a partial open question regarding topological indices: for which members of $n$-vertex graph family, certain index has minimum or maximum value? In this work, we answered the above-mentioned question regarding AZI and ABC for transformed families of graphs $\Gamma_{n}^{k, l}$ and $A_{\alpha}\left(\Gamma_{n}^{k, l}\right)$. We investigated the fact of pendent paths and the transformation $A_{\alpha}$ over these indices and developed the tight upper bounds regarding these families of graphs. Moreover, we characterized transformed graphs associated with maximum values of these indices.
\end{abstract}

\section{Introduction}

Nowadays, graph theory has potential applications in different fields of science. It is especially used for theoretical study of chemical compounds in chemistry. This area of study named as chemical graph theory deals with the problems related to the properties in chemistry. In the middle of last century, theoretical study of chemical compounds attracted the researchers due to its effective applications such as prediction of physiochemical properties of substances in cheminformatics, pharmaceutical sciences, materials science, engineering, and so forth [1]. Cheminformatics is comparatively the latest area of information technology which comprises chemistry, mathematics, and other informational sciences that concentrate on gathering, storing, treating, and examining chemical data. There are many theoretical molecular descriptors in literature used to predict properties of chemical compounds. Among these molecular descriptors, topological indices have an impact in chemistry due to the prediction of physiochemical properties of underlying substance. Its role in "quantitative-structure property relationship" (QSPR) or "quantitative-structure activity relationship" (QSAR) investigation models is also remarkable $[2,3]$.

In 1947, Wiener for the first time introduced the use of topological index during his work on paraffin's boiling points [4] and provided that it has best correlation with the boiling points of alkanes. The discovery of the Wiener index provided emerging research platform to the research community. In the later years, researchers of different communities proposed many other topological indices and used them for approximation of the chemical properties of their own interest.

In the race for better prediction, Randic [5] in 1975 introduced degree-based topological index named Randić connectivity index which was the best predictive invariant in 
those days. The Randić index was reported as the first degree-based index in QSPR study because Zagreb indices by Gutman and Trinajstic [6] were used for totally different purpose before Randić index. In 1998, parallel to the work of Bollobás and Erdös [7], Estrada et al. [8] defined atom bond connectivity $(\mathrm{ABC})$ index as

$$
\operatorname{ABC}(\Gamma)=\sum_{u v \in E(\Gamma)} \sqrt{\frac{\operatorname{deg}_{u}+\operatorname{deg}_{v}-2}{\operatorname{deg}_{u} \times \operatorname{deg}_{v}}}
$$

which has a good correlation with the heat of formation of alkanes. Star graph among trees and complete graph in general for fixed number of vertices have maximal value for $\mathrm{ABC}$ index [9]. For more details, one can see [10, 11]. Furtula et al. [12] made a generalization of $\mathrm{ABC}$ index as

$$
\operatorname{ABC}_{\lambda}(\Gamma)=\sum_{u v \in E(\Gamma)}\left(\frac{\operatorname{deg}_{u}+\operatorname{deg}_{v}-2}{\operatorname{deg}_{u} \times \operatorname{deg}_{v}}\right)^{-\lambda}
$$

by replacing $1 / 2$ with $-\lambda$. The augmented Zagreb index AZI is $\mathrm{ABC}_{\lambda}, \lambda=3$ as,

$$
\operatorname{AZI}(\Gamma)=\sum_{u v \in E(\Gamma)}\left(\frac{\operatorname{deg}_{u} \times \operatorname{deg}_{v}}{\operatorname{deg}_{u}+\operatorname{deg}_{v}-2}\right)^{3}
$$

Its correlation potential reported is even better than that of other indices in [13-15].

Mathematical study of ABC index and AZI [16-35] encouraged us to answer the fundamental question regarding characterization of transformed families of graphs with maximum and minimum values for $A B C$ index and
AZI. Gupta et.al. determined bounds for symmetric division degree index in [36]. In this work, we studied ABC index and AZI for transformed graphs $\Gamma_{n}^{k, l}$ and $A_{\alpha}\left(\Gamma_{n}^{k, l}\right)$ under the fact of transformation $A_{\alpha}, 0 \leq \alpha \leq l-2$. We characterize extremal graphs of these transformed families of graphs for AZI and ABC and established their bounds for $\Gamma_{n}^{k, l}$ and $A_{\alpha}\left(\Gamma_{n}^{k, l}\right)$. When a path is attached with the fully connected vertex (vertex with degree greater than one) of the graph, then it has an impact over the increase and decrease of the index under study. Throughout this work, consider graph $\Gamma_{n}^{k, l}[37]$. It comprises $n$-vertex simple connected graph $\Gamma$ along with $k$ pendent paths of length $l \geq 2$ attached with $v \in \Gamma$ having degree $2 \leq d_{v} \leq \Delta_{\Gamma}$. Let $\operatorname{deg}_{u_{1}}=\delta_{\Gamma} \leq \operatorname{deg}_{u_{2}} \leq$ $\operatorname{deg}_{u_{3}} \leq \ldots \leq \Delta_{\Gamma}+1$ be the degree sequence of $\Gamma_{n}^{k, l} . \Gamma_{n}^{k, l^{2}}$ is shown in Figure 1.

1.1. Graph Transformations. Let $H(\Gamma) \subset E(\Gamma), \Gamma \prime=\Gamma-H$ be the new graph generated by removing set edges of $H(\Gamma)$, and $\Gamma \prime \prime=\Gamma-V_{1}(\Gamma)$ be the new graph generated by deleting set of vertices $V_{1}(\Gamma) \subset V(\Gamma)$. We define following transformations.

Let $A_{\alpha} ; 0 \leq \alpha \leq l-2$ be the transformation defined over pendent paths attached with the graph [38]. $A_{\alpha}$ has solid effect over increase and decrease of AZI and ABC.

1.1.1. Transformation A. Let $w_{j} \in V(\Gamma), \operatorname{deg}_{w_{j}} \geq 2$, for $1 \leq j \leq k \leq n$ and paths pendent at $w_{j}$ of the form $\left\{w_{j} u_{j}^{1}, u_{j}^{1} u_{j}^{2}, u_{j}^{2} u_{j}^{3}, \ldots, u_{j}^{l-1} u_{j}^{l}\right\}$ comprise $\Gamma_{n}^{k, l}$. Then,

$$
A\left(\Gamma_{n}^{k, l}\right)=\Gamma_{n}^{k, l}-\sum_{j=1}^{k}\left\{u_{j}^{2} u_{j}^{3}, u_{j}^{3} u_{j}^{4}, \ldots, u_{j}^{l-1} u_{j}^{l}\right\}+\sum_{j=1}^{k}\left\{w_{j} u_{j}^{2}, u_{j}^{2} u_{j}^{3}, \ldots, u_{j}^{l-1} u_{j}^{l}\right\}
$$

The transformation $A$ is shown in Figure 2.

1.1.2. Transformation $A_{\alpha} . A_{\alpha}$ is the $\alpha \geq 0$ time repetition of transformation $A$.

Let graph $\Gamma=\Gamma(V, E)$ with degree of vertex $u \in \Gamma, \delta_{\Gamma} \leq$ $\operatorname{deg}_{u} \leq \Delta_{\Gamma}$ and $\delta_{\Gamma} \leq \operatorname{deg}_{v} \leq \Delta_{\Gamma}+1$ be the degree of $v \in \Gamma_{n}^{k, l}$.

\section{Upper Bounds for $\operatorname{AZI}\left(\Gamma_{n}^{\mathrm{k}, \mathrm{l}}\right)$ and $\operatorname{AZI}\left(A_{\boldsymbol{\alpha}}\left(\Gamma_{n}^{k, l}\right)\right)$}

Initially, we proved Proposition 1, which is helpful to prove the main results for AZI.
Proposition 1. Let

$$
\begin{aligned}
f(\eta, \zeta) & =f(\zeta, \eta) \\
& =\left(\frac{\eta \zeta}{\eta+\zeta-2}\right)^{3} .
\end{aligned}
$$

Proof. Let

$$
\begin{aligned}
f(\eta, \zeta) & =f(\zeta, \eta) \\
& =\left(\frac{\eta \zeta}{\eta+\zeta-2}\right)^{3}
\end{aligned}
$$



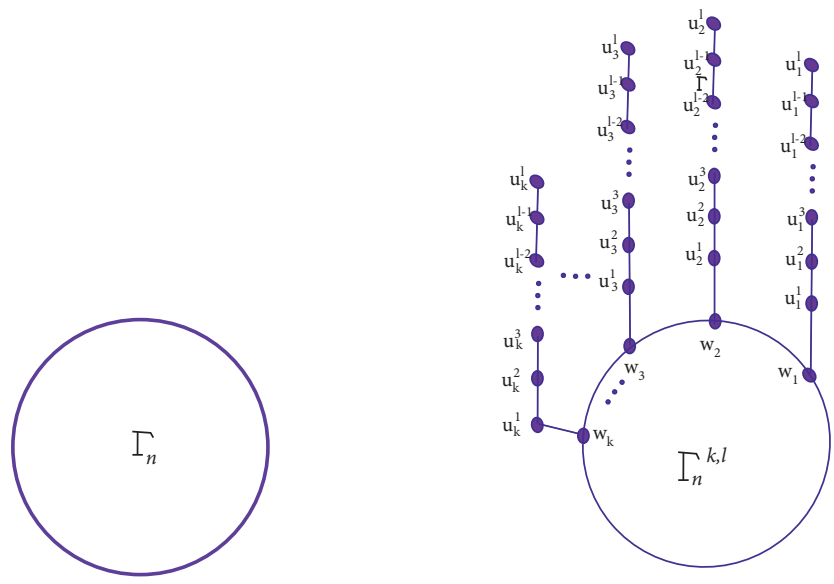

Figure 1: Graph $\Gamma_{n}^{k, l}$.

$$
\begin{aligned}
f(a, \zeta)-f(b, \zeta) & =\left(\frac{a \zeta}{a+\zeta-2}\right)^{3}-\left(\frac{b \zeta}{b+\zeta-2}\right)^{3} \\
& =\frac{\left(a^{3} \zeta^{3}(b+\zeta-2)^{3}\right)-b^{3} \zeta^{3}(a+\zeta-2)^{3}}{(a+\zeta-2)^{3}(b+\zeta-2)^{3}} \\
& =\frac{\zeta^{3}\left(a^{3}\left(b^{3}+(\zeta-2)^{3}+3 b^{2}(\zeta-2)+3 b(\zeta-2)^{2}\right)-b^{3}\left(a^{3}+(\zeta-2)^{3}+3 a^{2}(\zeta-2)+3 a(\zeta-2)^{2}\right)\right)}{(a+\zeta-2)^{3}(b+\zeta-2)^{3}} \\
& =\frac{\zeta^{3}\left(\left(a^{3}-b^{3}\right)(\zeta-2)^{3}+3 a^{2} b^{2}(a-b)(\eta-2)+3 a b\left(a^{2}-b^{2}\right)(\eta-2)^{2}\right)}{(a+\zeta-2)^{3}(b+\zeta-2)^{3}} \\
& =\frac{\zeta^{3}\left((a-b)\left(a^{2}+a b+b^{2}\right)(\zeta-2)^{3}+3 a^{2} b^{2}(a-b)(\zeta-2)+3 a b(a+b)(a-b)(\zeta-2)^{2}\right)}{(a+\zeta-2)^{3}(b+\zeta-2)^{3}} \\
& =\frac{\zeta^{3}(a-b)\left(\left(a^{2}+a b+b^{2}\right)(\zeta-2)^{3}+3 a^{2} b^{2}(\zeta-2)+3 a b(a+b)(\zeta-2)^{2}\right)}{(a+\zeta-2)^{3}(b+\zeta-2)^{3}} \geq 0 .
\end{aligned}
$$

This implies

$$
f(a, \zeta) \geq f(b, \zeta)
$$

Lemma 1 (see [15]). Let

$$
\begin{aligned}
\Phi(u, v) & =\Phi(v, u) \\
& =\left(\frac{\mathrm{uv}}{u+v-2}\right)^{3} .
\end{aligned}
$$

Then,
(1) $\Phi(1, v)$ is decreasing for $v \geq 2$.

(2) $\Phi(2, v)=8$ for any real number $v$.

(3) For fixed $v \geq 3, \Phi(u, v)$ is increasing and $\Phi(u, v)>8$ for $u>2$.

In Theorem 1, we discuss the effect of pendent paths over $A Z I$ and determine its upper bound.

Theorem 1. Let graph $\Gamma_{n}^{k, l}$ comprise $n$-vertex graph $\Gamma$ having $m$ edges and $p \geq 0$ pendent vertices. Then,

$$
\operatorname{AZI}\left(\Gamma_{n}^{k, l}\right) \leq k \frac{\Delta_{\Gamma}}{2}\left[\left(\frac{\left(\Delta_{\Gamma}+1\right)^{2}}{2 \Delta_{\Gamma}}\right)^{3}+\left(\frac{\Delta_{\Gamma}\left(\Delta_{\Gamma}+1\right)}{2 \Delta_{\Gamma}-1}\right)^{3}\right]+\mathrm{AZI}(\Gamma)-k \frac{\delta_{\Gamma}}{2}\left[\left(\frac{\delta_{\Gamma}\left(\delta_{\Gamma}+1\right)}{2 \delta_{\Gamma}-1}\right)^{3}+\left(\frac{\delta_{\Gamma}^{2}}{2 \delta_{\Gamma}-2}\right)^{3}\right]+8 \mathrm{kl}+p\left[8-\left(\frac{\Delta_{\Gamma}+1}{\Delta_{\Gamma}}\right)^{3}\right]
$$




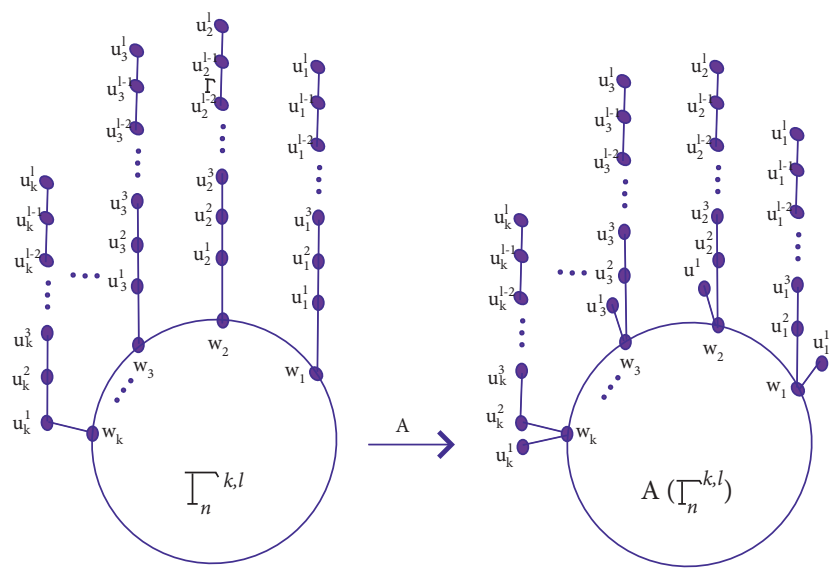

Figure 2: Transformation $A$.

Equality holds for a complete graph $\Gamma$ of size $n$ with pendent paths of length $l$ at each vertex, i.e., $k=n$.

Proof. Let $\Gamma_{n}^{k, l}$ be the graph formed by $k$ number of paths having length $l$ pendent at distinct vertices $u \in \Gamma$ such that $2 \leq \operatorname{deg}_{u} \leq \delta_{\Gamma}+1$. Then,

$$
\operatorname{AZI}(\Gamma)=\sum_{u v \in E(\Gamma)}\left(\frac{\operatorname{deg}_{u} \operatorname{deg}_{v}}{\operatorname{deg}_{u}+\operatorname{deg}_{v}-2}\right)^{3} .
$$

The construction of $\Gamma_{n}^{k, l}, l \geq 2$, implies $\left|E\left(\Gamma_{n}^{k, l}\right)\right|=m+\mathrm{kl}$, and for

$$
\begin{aligned}
\text { uv } & \in E\left(\Gamma_{n}^{k, l}\right), \\
\left(\operatorname{deg}_{u}+\operatorname{deg}_{v}\right) & \in\left\{3,4, \operatorname{deg}_{u}+3, \operatorname{deg}_{u}+\operatorname{deg}_{v}, \operatorname{deg}_{u}+\operatorname{deg}_{v}+1\right\} .
\end{aligned}
$$

$$
\begin{aligned}
& A_{3}=\left\{\mathrm{uv} \in \Gamma_{n}^{k, l}: \operatorname{deg}_{u}=1, \operatorname{deg}_{v}=2\right\}, \\
& A_{4}=\left\{\mathrm{uv} \in \Gamma_{n}^{k, l}: \operatorname{deg}_{u}=\operatorname{deg}_{v}=2\right\} \text {, } \\
& A_{\operatorname{deg}_{u}+3}=\left\{\mathrm{uv} \in \Gamma_{n}^{k, l}: \delta_{\Gamma} \leq \operatorname{deg}_{u}=\operatorname{deg}_{u} \leq \Delta_{\Gamma}+1, \operatorname{deg}_{v}=2\right\}, \\
& A_{\mathrm{deg}_{u}+\operatorname{deg}_{v}}=\left\{\mathrm{uv} \in \Gamma_{n}^{k, l}: \delta_{\Gamma} \leq \operatorname{deg}_{u}=\operatorname{deg}_{u}, \operatorname{deg}_{v}=\operatorname{deg}_{v} \leq \Delta_{\Gamma}\right\}, \\
& A_{\operatorname{deg}_{u}+\operatorname{deg}_{v}+1}=\left\{\mathrm{uv} \in \Gamma_{n}^{k, l}: \delta_{\Gamma} \leq \operatorname{deg}_{u}=\operatorname{deg}_{u}, \operatorname{deg}_{v}=\operatorname{deg}_{v}+1 \leq \Delta_{\Gamma}+1\right\}, \\
& \operatorname{AZI}\left(\Gamma_{n}^{k, l}\right)=\sum_{\text {uv are edges of pendent paths }}\left(\frac{\operatorname{deg}_{u} \operatorname{deg}_{v}}{\operatorname{deg}_{u}+\operatorname{deg}_{v}-2}\right)^{3}+\sum_{\text {uv are edges of } \Gamma}\left(\frac{\operatorname{deg}_{u} \operatorname{deg}_{v}}{\operatorname{deg}_{u}+\operatorname{deg}_{v}-2}\right)^{3}, \\
& \operatorname{AZI}\left(\Gamma_{n}^{k, l}\right)=\sum_{u v \in A_{3}}\left(\frac{\operatorname{deg}_{u} \operatorname{deg}_{v}}{\operatorname{deg}_{u}+\operatorname{deg}_{v}-2}\right)^{3}+\sum_{u v \in A_{4}}\left(\frac{\operatorname{deg}_{u} \operatorname{deg}_{v}}{\operatorname{leg}_{u}+\operatorname{deg}_{v}-2}\right)^{3}+\sum_{u v \in A_{\operatorname{deg}_{u}+3}}\left(\frac{\operatorname{deg}_{u} \operatorname{deg}_{v}}{\operatorname{leg}_{u}+\operatorname{deg}_{v}-2}\right)^{3}+\sum_{u v \in A} \sum_{\operatorname{deg}_{u}+\operatorname{deg}_{u}+1}\left(\frac{\operatorname{deg}_{u} \operatorname{deg}_{v}}{\operatorname{deg}_{u}+\operatorname{deg}_{v}-2}\right)^{3} \\
& \operatorname{deg}_{u}, \operatorname{deg}_{u} \geq 2
\end{aligned}
$$

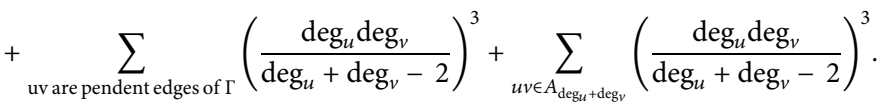


The construction of AZI $\left(\Gamma_{n}^{k, l}\right)$ implies that the cardinality of $A_{3} \quad$ is $\quad k$, i.e., $\left|A_{3}\right|=k,\left|A_{4}\right|=k(l-2), \quad\left|A_{\operatorname{deg}_{u}+3}\right|=k$, $\left|A_{\operatorname{deg}_{u}+3}\right|=k,\left|\begin{array}{c}A \operatorname{deg}_{u}+\operatorname{deg}_{u}+1 \\ \operatorname{deg}_{u}, \operatorname{deg}_{u} \geq 2\end{array}\right| \leq k \Delta_{\Gamma}-p, \quad$ and

$$
\begin{aligned}
& \sum_{\text {uv are pendentedges of } \Gamma}\left(\frac{\operatorname{deg}_{u} \operatorname{deg}_{v}}{\operatorname{deg}_{u}+\operatorname{deg}_{v}-2}\right)^{3} \leq 8 p, \\
& \sum_{\substack{u v \in A \\
\operatorname{deg}_{u}+\operatorname{deg}_{u}+1 \\
\operatorname{deg}_{u}, \operatorname{leg}_{u} \geq 2}}\left(\frac{\operatorname{deg}_{u} \operatorname{deg}_{v}}{\operatorname{deg}_{u}+\operatorname{deg}_{v}-2}\right)^{3} \leq \frac{k \Delta_{\Gamma}}{2}\left[\left(\frac{\left(\Delta_{\Gamma}+1\right)^{2}}{2 \Delta_{\Gamma}}\right)^{3}+\left(\frac{\Delta_{\Gamma}\left(\Delta_{\Gamma}+1\right)}{2 \Delta_{\Gamma}-1}\right)^{3}\right], \\
& \sum_{\substack{u v \in A \\
\operatorname{deg}_{u}+\operatorname{deg}_{u} \\
\operatorname{deg}_{u}, \operatorname{leg}_{u} \geq 2}}\left(\frac{\operatorname{deg}_{u} \operatorname{deg}_{v}}{\operatorname{deg}_{u}+\operatorname{deg}_{v}-2}\right)^{3} \leq \operatorname{AZI}(\Gamma)-p\left(\frac{\Delta_{\Gamma}+1}{\Delta_{\Gamma}}\right)^{3}-\frac{k \delta_{\Gamma}}{2}\left[\left(\frac{\delta_{\Gamma}\left(\delta_{\Gamma}+1\right)}{2 \delta_{\Gamma}-1}\right)^{3}+\left(\frac{\delta_{\Gamma}^{2}}{2 \delta_{\Gamma}-2}\right)^{3}\right] .
\end{aligned}
$$

Now, from equation (12), we get

$$
\begin{aligned}
\operatorname{AZI}\left(\Gamma_{n}^{k, l}\right) \leq & k \frac{\Delta_{\Gamma}}{2}\left[\left(\frac{\left(\Delta_{\Gamma}+1\right)^{2}}{2 \Delta_{\Gamma}}\right)^{3}+\left(\frac{\Delta_{\Gamma}\left(\Delta_{\Gamma}+1\right)}{2 \Delta_{\Gamma}-1}\right)^{3}\right]+\mathrm{AZI}(\Gamma)-k \frac{\delta_{\Gamma}}{2}\left[\left(\frac{\delta_{\Gamma}\left(\delta_{\Gamma}+1\right)}{2 \delta_{\Gamma}-1}\right)^{3}+\left(\frac{\delta_{\Gamma}^{2}}{2 \delta_{\Gamma}-2}\right)^{3}\right] \\
& -p\left(\frac{\Delta_{\Gamma}+1}{\Delta_{\Gamma}}\right)^{3}+8 k+8 k(l-2)+8 k+8 p .
\end{aligned}
$$

After simplification, we get

$$
\operatorname{AZI}\left(\Gamma_{n}^{k, l}\right) \leq k \frac{\Delta_{\Gamma}}{2}\left[\left(\frac{\left(\Delta_{\Gamma}+1\right)^{2}}{2 \Delta_{\Gamma}}\right)^{3}+\left(\frac{\Delta_{\Gamma}\left(\Delta_{\Gamma}+1\right)}{2 \Delta_{\Gamma}-1}\right)^{3}\right]+\operatorname{AZI}(\Gamma)-k \frac{\delta_{\Gamma}}{2}\left[\left(\frac{\delta_{\Gamma}\left(\delta_{\Gamma}+1\right)}{2 \delta_{\Gamma}-1}\right)^{3}+\left(\frac{\delta_{\Gamma}^{2}}{2 \delta_{\Gamma}-2}\right)^{3}\right]+8 \mathrm{kl}+p\left[8-\left(\frac{\Delta_{\Gamma}+1}{\Delta_{\Gamma}}\right)^{3}\right] .
$$

Inequality (15) completes the proof.

In Theorem 2, we discussed the effect of successive applications of transformation $A$ as shown in Figure 2 over AZI.
Theorem 2. Let graph $\Gamma_{n}^{k, l}$ comprise $n$-vertex simple connected graph $\Gamma$. Then,

$$
\begin{aligned}
\operatorname{AZI}\left(A_{\alpha}\left(\Gamma_{n}^{k, l}\right)\right) \leq & \frac{k \Delta_{\Gamma}}{2}\left[\left(\frac{\left(\Delta_{\Gamma}+\alpha+1\right)^{2}}{2 \Delta_{\Gamma}+\alpha}\right)^{3}+\left(\frac{\Delta_{\Gamma}\left(\Delta_{\Gamma}+\alpha+1\right)}{2 \Delta_{\Gamma}+\alpha-1}\right)^{3}\right]-\frac{k \delta_{\Gamma}}{2}\left[\left(\frac{\delta_{\Gamma}\left(\delta_{\Gamma}+\alpha+1\right)}{2 \delta_{\Gamma}+\alpha-1}\right)^{3}+\left(\frac{\delta_{\Gamma}^{2}}{2 \delta_{\Gamma}-2}\right)^{3}\right]+p\left[8-\left(\frac{\Delta_{\Gamma}+1}{\Delta_{\Gamma}}\right)^{3}\right] \\
& +8 \mathrm{kl}-8 k \alpha+\operatorname{AZI}(\Gamma) .
\end{aligned}
$$


Equality holds for a complete graph $\Gamma$ of size $n$ with pendent paths of length $l$ at each vertex, i.e., $k=n$.

Proof. Let a simple graph $\Gamma$ of order $n$, Size $m$ having $p \geq 0$ pendent vrtives. The augmented Zagreb index of any graph $\Gamma$ is

$$
\operatorname{AZI}(\Gamma)=\sum_{u v \in E(\Gamma)}\left(\frac{\operatorname{deg}_{u} \operatorname{deg}_{v}}{\operatorname{deg}_{u}+\operatorname{deg}_{v}-2}\right)^{3}
$$

$$
\left(\operatorname{deg}_{u}+\operatorname{deg}_{v}\right) \in\left\{3,4, \operatorname{deg}_{u}+\alpha+2, \operatorname{deg}_{u}+\alpha+3, \operatorname{deg}_{u}+\operatorname{deg}_{v}, \operatorname{deg}_{u}+\alpha+1+\operatorname{deg}_{v}\right\},
$$

which implies

$$
\begin{aligned}
& E_{3}\left(A_{\alpha}\left(\Gamma_{n}^{k, l}\right)\right)=\left\{\mathrm{uv} \in \Gamma_{n}^{k, l}: \operatorname{deg}_{u}=1, \operatorname{deg}_{v}=2\right\}, \\
& E_{4}\left(A_{\alpha}\left(\Gamma_{n}^{k, l}\right)\right)=\left\{\mathrm{uv} \in \Gamma_{n}^{k, l}: \operatorname{deg}_{u}=\operatorname{deg}_{v}=2\right\}, \\
& E_{\operatorname{deg}_{u}+\alpha+2}\left(A_{\alpha}\left(\Gamma_{n}^{k, l}\right)\right)=\left\{\mathrm{uv} \in \Gamma_{n}^{k, l}: \delta_{\Gamma} \leq \operatorname{deg}_{u}=\operatorname{deg}_{u}+\alpha+1 \leq \Delta_{\Gamma}+\alpha+1, \operatorname{deg}_{v}=1\right\}, \\
& E_{\operatorname{deg}_{u}+\alpha+3}\left(A_{\alpha}\left(\Gamma_{n}^{k, l}\right)\right)=\left\{\mathrm{uv} \in \Gamma_{n}^{k, l}: \delta_{\Gamma} \leq \operatorname{deg}_{u}=\operatorname{deg}_{u}+\alpha+1 \leq \Delta_{\Gamma}+\alpha+1, \operatorname{deg}_{v}=2\right\}, \\
& E_{\operatorname{deg}_{u}+\operatorname{deg}_{v}}\left(A_{\alpha}\left(\Gamma_{n}^{k, l}\right)\right)=\left\{\mathrm{uv} \in \Gamma_{n}^{k, l}: \delta_{\Gamma} \leq \operatorname{deg}_{u}=\operatorname{deg}_{u}, \operatorname{deg}_{v}=\operatorname{deg}_{v} \leq \Delta_{\Gamma}\right\}, \\
& E_{\operatorname{deg}_{u}+\alpha+1+\operatorname{deg}_{v}}\left(A_{\alpha}\left(\Gamma_{n}^{k, l}\right)\right)=\left\{\mathrm{uv} \in \Gamma_{n}^{k, l}: \delta_{\Gamma} \leq \operatorname{deg}_{v}=\operatorname{deg}_{v}, \operatorname{deg}_{u} \leq \Delta_{\Gamma}, \operatorname{deg}_{u}=\operatorname{deg}_{u}+\alpha+1\right\}, \\
& \operatorname{AZI}\left(A_{\alpha}\left(\Gamma_{n}^{k, l}\right)\right)=\sum_{\text {uv are edges of pendent paths }}\left(\frac{\operatorname{deg}_{u} \operatorname{deg}_{v}}{\operatorname{deg}_{u}+\operatorname{deg}_{v}-2}\right)^{3}+\sum_{\text {uv are edges of } \Gamma}\left(\frac{\operatorname{deg}_{u} \operatorname{deg}_{v}}{\operatorname{deg}_{u}+\operatorname{deg}_{v}-2}\right)^{3}, \\
& \operatorname{AZI}\left(A_{\alpha}\left(\Gamma_{n}^{k, l}\right)\right)=\sum_{u v \in A_{3}}\left(\frac{\operatorname{deg}_{u} \operatorname{deg}_{v}}{\operatorname{deg}_{u}+\operatorname{deg}_{v}-2}\right)^{3}+\sum_{u v \in A_{4}}\left(\frac{\operatorname{deg}_{u} \operatorname{deg}_{v}}{\operatorname{deg}_{u}+\operatorname{deg}_{v}-2}\right)^{3}+\sum_{u v \in A_{\operatorname{deg}_{u}+\alpha+2}}\left(\frac{\operatorname{deg}_{u} \operatorname{deg}_{v}}{\operatorname{leg}_{u}+\operatorname{deg}_{v}-2}\right)^{3}+\sum_{u v \in A_{\operatorname{deg}_{u}+\alpha+3}}\left(\frac{\operatorname{deg}_{u} \operatorname{deg}_{v}}{\operatorname{deg}_{u}+\operatorname{deg}_{v}-2}\right)^{3}
\end{aligned}
$$

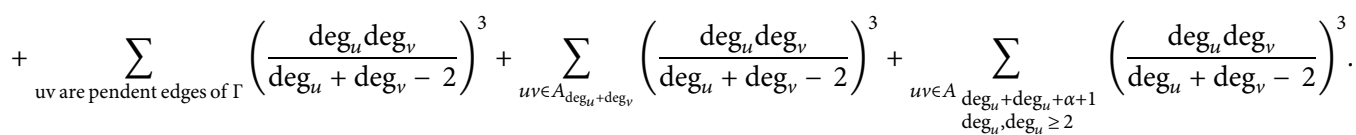
is

The construction of AZI $\left(\Gamma_{n}^{k, l}\right)$ implies that the cardinality

$$
\begin{aligned}
\left|E_{3}\left(A_{\alpha}\left(\Gamma_{n}^{k, l}\right)\right)\right| & =k, \\
\left|E_{4}\left(A_{\alpha}\left(\Gamma_{n}^{k, l}\right)\right)\right| & =k(l-\alpha-2), \\
\left|E_{\operatorname{deg}_{u}+\alpha+2}\left(A_{\alpha}\left(\Gamma_{n}^{k, l}\right)\right)\right| & =k \alpha,
\end{aligned}
$$

$$
\begin{gathered}
\left|E_{\operatorname{deg}_{u}+\alpha+3}\left(A_{\alpha}\left(\Gamma_{n}^{k, l}\right)\right)\right|=k, \\
\left|A_{\text {de } g_{u}+\text { de } g_{u}+1 \quad} \quad\right| \leq k \Delta_{\Gamma}-p, \\
\text { de } g_{u} \text {, de } g_{u} \quad \geq 2
\end{gathered}
$$

and $\left|A_{1+\operatorname{deg}_{u}}\right|=p$. For $\delta_{\Gamma}$ minimum degree of vertices of $\Gamma$ and maximum degree $\Delta_{\Gamma}$, using Lemma 1 , we have 


$$
\begin{aligned}
& \sum_{\text {uv are pendent edges of } \Gamma}\left(\frac{\operatorname{deg}_{u} \operatorname{deg}_{v}}{\operatorname{leg}_{u}+\operatorname{deg}_{v}-2}\right)^{3} \leq 8 p, \\
& \sum_{\substack{u v A \\
\operatorname{deg}_{u}+\operatorname{deg}_{v}+\alpha+1 \\
\operatorname{deg}_{u}, \operatorname{deg}_{u} \geq 2}}\left(\frac{\operatorname{deg}_{u} \operatorname{deg}_{v}}{\operatorname{leg}_{u}+\operatorname{deg}_{v}-2}\right)^{3} \leq \frac{k \Delta_{\Gamma}}{2}\left[\left(\frac{\left(\Delta_{\Gamma}+\alpha+1\right)^{2}}{2 \Delta_{\Gamma}+2 \alpha}\right)^{3}+\left(\frac{\Delta_{\Gamma}\left(\Delta_{\Gamma}+\alpha+1\right)}{2 \Delta_{\Gamma}+\alpha-1}\right)^{3}\right],
\end{aligned}
$$

$$
\begin{aligned}
& \sum_{u v \in A_{\operatorname{deg}_{u}+\alpha+2}}\left(\frac{\operatorname{deg}_{u} \operatorname{deg}_{v}}{\operatorname{deg}_{u}+\operatorname{deg}_{v}-2}\right)^{3} \leq k \alpha\left(\frac{\Delta_{\Gamma}+\alpha+1}{\Delta_{\Gamma}+\alpha}\right)^{3}, \\
& \sum_{u v \in A_{\operatorname{deg}_{u}+\alpha+3}}\left(\frac{\operatorname{deg}_{u} \operatorname{deg}_{v}}{\operatorname{deg}_{u}+\operatorname{deg}_{v}-2}\right)^{3} \leq 8 k .
\end{aligned}
$$

Substituting these changes in equation (19), we have following inequality.

$$
\begin{aligned}
\operatorname{AZI}\left(A_{\alpha}\left(\Gamma_{n}^{k, l}\right)\right) \leq & 8 k+8 k(l-2-\alpha)+8 k+k \alpha\left(\frac{\Delta_{\Gamma}+\alpha+1}{\Delta_{\Gamma}+\alpha}\right)^{3}+8 p+k \frac{\Delta_{\Gamma}}{2}\left[\left(\frac{\left(\Delta_{\Gamma}+\alpha+1\right)^{2}}{2 \Delta_{\Gamma}+\alpha}\right)^{3}+\left(\frac{\Delta_{\Gamma}\left(\Delta_{\Gamma}+\alpha+1\right)}{2 \Delta_{\Gamma}+\alpha-1}\right)^{3}\right] \\
& +\operatorname{AZI}(\Gamma)-k \frac{\delta_{\Gamma}}{2}\left[\left(\frac{\delta_{\Gamma}\left(\delta_{\Gamma}+\alpha+1\right)}{2 \delta_{\Gamma}+\alpha-1}\right)^{3}+\left(\frac{\delta_{\Gamma}^{2}}{2 \delta_{\Gamma}-2}\right)^{3}\right]-p\left(\frac{\Delta_{\Gamma}+\alpha+1}{\Delta_{\Gamma}+\alpha}\right)^{3} .
\end{aligned}
$$

After simplification, we get

$$
\begin{aligned}
\operatorname{AZI}\left(A_{\alpha}\left(\Gamma_{n}^{k, l}\right)\right) \leq & \frac{k \Delta_{\Gamma}}{2}\left[\left(\frac{\left(\Delta_{\Gamma}+\alpha+1\right)^{2}}{2 \Delta_{\Gamma}+\alpha}\right)^{3}+\left(\frac{\Delta_{\Gamma}\left(\Delta_{\Gamma}+\alpha+1\right)}{2 \Delta_{\Gamma}+\alpha-1}\right)^{3}\right]-\frac{k \delta_{\Gamma}}{2}\left[\left(\frac{\delta_{\Gamma}\left(\delta_{\Gamma}+\alpha+1\right)}{2 \delta_{\Gamma}+\alpha-1}\right)^{3}+\left(\frac{\delta_{\Gamma}^{2}}{2 \delta_{\Gamma}-2}\right)^{3}\right] \\
& +p\left[8-\left(\frac{\Delta_{\Gamma}+1}{\Delta_{\Gamma}}\right)^{3}\right]+8 \mathrm{kl}-8 k \alpha+\mathrm{AZI}(\Gamma) .
\end{aligned}
$$

Inequality (23) completes the proof.

\section{Upper Bounds for $\operatorname{ABC}\left(\Gamma_{n}^{k, l}\right)$ and $\mathbf{A B C}\left(A_{\boldsymbol{\alpha}}\left(\Gamma_{n}^{k, l}\right)\right)$}

Lemma 2 (see $[39,40])$. Let

$$
\begin{aligned}
\Phi(x, y) & =\Phi(y, x) \\
& =\sqrt{\frac{x+y-2}{x y}} .
\end{aligned}
$$

Then,

(1) $\Phi(1, y)$ is increasing for $y$.

(2) $\Phi(2, y)=\sqrt{2} / 2$ for any real number $y$.
(3) For fixed $y \geq 2, \Phi(x, y)$ is decreasing for $x$. Proposition 2 is related to the $\mathrm{ABC}$ index.

Proposition 2. Let

$$
\begin{aligned}
\Phi(\eta, \zeta) & =\Phi(\zeta, \eta) \\
& =\sqrt{\frac{\eta+\zeta-2}{\eta \zeta}} .
\end{aligned}
$$

For $\eta, \zeta, \Delta \in \mathfrak{R}, \eta, \zeta \leq \Delta$ and $\eta, \zeta, \Delta \geq 2$.

$$
\Phi(\eta, \zeta) \geq \Phi(\Delta, \Delta) \text {. }
$$




$$
\begin{aligned}
\Phi(\eta, \zeta) & =\Phi(\zeta, \eta) \\
& =\sqrt{\frac{\eta+\zeta-2}{\eta \zeta}}
\end{aligned}
$$

By Lemma 2, for $\eta=2$ or $\zeta=2$ and $\Delta=2$,

$$
\begin{aligned}
\Phi(\eta, \zeta) & =\Phi(\Delta, \Delta) \\
& =\Phi(\Delta, \zeta)
\end{aligned}
$$

$$
\begin{aligned}
& =\Phi(\eta, \Delta) \\
& =\frac{\sqrt{2}}{2} .
\end{aligned}
$$

Now for $\eta \geq 3$ or $\zeta \geq 3$ and $\Delta=\zeta$, let $\alpha, \beta, \gamma \geq 1, \alpha \leq \gamma, \beta=$ $\gamma$ and $\eta=2+\alpha$ or $\zeta=2+\beta, \Delta=2+\gamma$,

$$
\begin{aligned}
& \Phi(\eta, \zeta)-\Phi(\Delta, \Delta)=\sqrt{\frac{\eta+\zeta-2}{\eta \zeta}}-\sqrt{\frac{\Delta+\Delta-2}{\Delta \Delta}} \\
& =\sqrt{\frac{(\alpha+2)+(\beta+2)-2}{(\alpha+2)(\beta+2)}}-\sqrt{\frac{2(\gamma+2)-2}{(\gamma+2)^{2}}} \\
& =\sqrt{\frac{\alpha+2+\beta}{(\alpha+2)(\beta+2)}}-\sqrt{\frac{2(\gamma+2)-2}{(\gamma+2)^{2}}} \\
& =\frac{1}{\sqrt{\alpha+2+\beta /(\alpha+2)(\beta+2)}+\sqrt{2(\gamma+2)-2 /(\gamma+2)^{2}}}\left[\frac{\alpha+2+\beta}{(\alpha+2)(\beta+2)}-\frac{2(\gamma+2)-2}{(\gamma+2)^{2}}\right] \\
& =\frac{1}{\sqrt{\alpha+2+\beta /(\alpha+2)(\beta+2)}+\sqrt{2(\gamma+2)-2 /(\gamma+2)^{2}}}=\left[\frac{1}{(\beta+2)}+\frac{\beta}{(\alpha+2)(\beta+2)}-\frac{2}{(\gamma+2)}+\frac{2}{(\gamma+2)^{2}}\right] \\
& =\frac{1}{\sqrt{\alpha+2+\beta /(\alpha+2)(\beta+2)}+\sqrt{2(\gamma+2)-2 /(\gamma+2)^{2}}}\left[\frac{\gamma-\beta}{(\beta+2)}+\frac{\beta}{(\alpha+2)(\beta+2)}-\frac{1}{(\gamma+2)}+\frac{2}{(\gamma+2)^{2}}\right] \\
& =\frac{1}{\sqrt{\alpha+2+\beta /(\alpha+2)(\beta+2)}+\sqrt{2(\gamma+2)-2 /(\gamma+2)^{2}}}\left[\frac{\gamma-\beta}{(\beta+2)}+\beta /(\alpha+2)(\beta+2)-\gamma /(\gamma+2)^{2}\right] \\
& =\frac{1}{\sqrt{\alpha+2+\beta /(\alpha+2)(\beta+2)}+\sqrt{2(\gamma+2)-2 /(\gamma+2)^{2}}}\left[\frac{\gamma-\beta}{(\beta+2)}+\frac{\beta(\gamma+2)^{2}-\gamma(\alpha+2)(\beta+2)}{(\alpha+2)(\beta+2)(\gamma+2)^{2}}\right] \\
& =\frac{1}{\sqrt{\alpha+2+\beta /(\alpha+2)(\beta+2)}+\sqrt{2(\gamma+2)-2 /(\gamma+2)^{2}}}\left[\frac{\gamma-\beta}{(\beta+2)}+\frac{\beta \gamma(\gamma-\alpha)+2 \gamma(\beta-\alpha)+4 \beta-4 \gamma}{(\alpha+2)(\beta+2)(\gamma+2)^{2}}\right] \\
& =\frac{1}{\sqrt{\alpha+2+\beta /(\alpha+2)(\beta+2)}+\sqrt{2(\gamma+2)-2 /(\gamma+2)^{2}}}\left[\frac{\gamma-\beta}{(\beta+2)}+\frac{\beta \gamma(\gamma-\alpha)+2 \gamma(\beta-\alpha)+4(\beta-\gamma)}{(\alpha+2)(\beta+2)(\gamma+2)^{2}}\right] \\
& =\frac{1}{\sqrt{\alpha+2+\beta /(\alpha+2)(\beta+2)}+\sqrt{2(\gamma+2)-2 /(\gamma+2)^{2}}}\left[\frac{\beta \gamma(\gamma-\alpha)+2 \gamma(\beta-\alpha)}{(\alpha+2)(\beta+2)(\gamma+2)^{2}}+\frac{(\gamma-\beta)\left((\alpha+2)(\gamma+2)^{2}-4\right)}{(\alpha+2)(\beta+2)(\gamma+2)^{2}}\right] \\
& =\frac{1}{\sqrt{\eta+\zeta-2 / \eta \zeta}+\sqrt{\Delta+\Delta-2 / \Delta \Delta}}\left[\frac{(\Delta-2)((\zeta-2)(\Delta-\eta)+2(\zeta-\eta))}{\eta \zeta \Delta^{2}}+\frac{(\Delta-\zeta)\left(\eta \Delta^{2}-4\right)}{\eta \zeta \Delta^{2}}\right] .
\end{aligned}
$$


Since $\Phi(\eta, \zeta)$ is a symmetric function, one can let $\eta \geq \zeta$ or $\zeta \geq \eta$, so the factor $(\zeta-\eta) \geq 0$ along with $\eta-2 \geq 0, \zeta-2 \geq 0$, $\Delta-2 \geq 0 \Delta-\eta \geq 0$, and $\Delta-\zeta \geq 0$. All the factors involved in equation (29) are positive. This implies

$$
\Phi(\eta, \zeta)-\Phi(\Delta, \Delta) \geq 0 .
$$

Hence, for all $\eta, \zeta \geq 2$ and $\eta, \zeta \leq \Delta$,

$$
\Phi(\eta, \zeta) \geq \Phi(\Delta, \Delta) .
$$

In Theorem 3, we discuss the effect of pendent paths over $\mathrm{ABC}$ index and determine its upper bound.

Theorem 3. Let graph $\Gamma_{n}^{k, l}$ and $\Gamma$ having order $n$, size $m$, and $p \geq 0$ pendent vertices. Then,

$$
\operatorname{ABC}\left(\Gamma_{n}^{k, l}\right) \leq k \Delta_{\Gamma} \sqrt{\frac{1}{2}}-\frac{k \delta_{\Gamma}}{2}\left[\sqrt{\frac{2 \Delta_{\Gamma}}{\left(\Delta_{\Gamma}+1\right)^{2}}}+\sqrt{\frac{2 \Delta_{\Gamma}-1}{\Delta_{\Gamma}\left(\Delta_{\Gamma}+1\right)}}\right]+\frac{\sqrt{2}}{2} \mathrm{kl}+p\left(\sqrt{\frac{\Delta_{\Gamma}}{\left(\Delta_{\Gamma}+1\right)}}-\frac{\sqrt{2}}{2}\right)+\operatorname{ABC}(\Gamma) .
$$

Equality holds for $\Gamma=C_{n}$ with pendent paths at each vertex, i.e., $k=n$.

Proof. Let $\Gamma_{n}^{k, l}$ be the graph. $\mathrm{ABC}(\Gamma)$ is

$$
\operatorname{ABC}(\Gamma)=\sum_{u v \in E(\Gamma)} \sqrt{\frac{\operatorname{deg}_{u}+\operatorname{deg}_{v}-2}{\operatorname{deg}_{u} \operatorname{deg}_{v}}} .
$$

The construction of $\Gamma_{n}^{k, l}, l \geq 2$, implies $\left|E\left(\Gamma_{n}^{k, l}\right)\right|=m+k l$, and for

$$
\mathrm{uv} \in E\left(\Gamma_{n}^{k, l}\right),
$$

$$
\left(\operatorname{deg}_{u}+\operatorname{deg}_{v}\right) \in\left\{3,4, \operatorname{deg}_{u}+3, \operatorname{deg}_{u}+\operatorname{deg}_{v}, \operatorname{deg}_{u}+\operatorname{deg}_{v}+1\right\} \text {. }
$$

We use edge set partition of $\Gamma_{n}^{k, l}$ defined in Theorem 1:

$$
\begin{aligned}
\operatorname{ABC}\left(\Gamma_{n}^{k, l}\right)= & \sum_{\text {uv are edges of pendent paths }} \sqrt{\frac{\operatorname{deg}_{u}+\operatorname{deg}_{v}-2}{\operatorname{deg}_{u} \operatorname{deg}_{v}}}+\sum_{\text {uv are edges of } \Gamma} \sqrt{\frac{\operatorname{deg}_{u}+\operatorname{deg}_{v}-2}{\operatorname{deg}_{u} \operatorname{deg}_{v}}}, \\
\operatorname{ABC}\left(\Gamma_{n}^{k, l}\right)= & \sum_{u v \in A_{3}} \sqrt{\frac{\operatorname{deg}_{u}+\operatorname{deg}_{v}-2}{\operatorname{deg}_{u} \operatorname{deg}_{v}}}+\sum_{u v \in A_{4}} \sqrt{\frac{\operatorname{deg}_{u}+\operatorname{deg}_{v}-2}{\operatorname{deg}_{u} \operatorname{deg}_{v}}}+\sum_{u v \in A_{\operatorname{deg}_{u}+3}} \sqrt{\frac{\operatorname{deg}_{u}+\operatorname{deg}_{v}-2}{\operatorname{deg}_{u} \operatorname{deg}_{v}}} \\
& +\sum_{u v \in A} \sum_{\operatorname{deg}_{u}+\operatorname{deg}_{u}+1} \sqrt{\frac{\operatorname{deg}_{u}+\operatorname{deg}_{v}-2}{\operatorname{deg}_{u} \operatorname{deg}_{v}}}+\sum_{\text {uv are pendent edges of } \Gamma} \sqrt{\frac{\operatorname{deg}_{u}+\operatorname{deg}_{v}-2}{\operatorname{deg}_{u} \operatorname{deg}_{v}}}+\sum_{u v \in A_{\operatorname{deg}_{u}+\operatorname{leg}_{v}}} \sqrt{\frac{\operatorname{deg}_{u}+\operatorname{deg}_{v}-2}{\operatorname{deg}_{u} \operatorname{deg}_{v}}} . \\
& \operatorname{deg}_{u}, \operatorname{deg}_{u} \geq 2
\end{aligned}
$$

The construction of $\mathrm{ABC}\left(\Gamma_{n}^{k, l}\right)$ implies that the cardinality of $A_{3}$ is $k$, i.e., $\left|A_{3}\right|=k,\left|A_{4}\right|=k(l-2),\left|A_{\operatorname{deg}_{u}+3}\right|=k$,

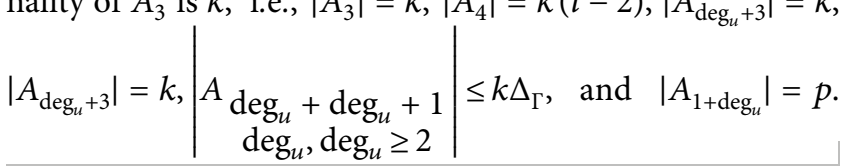

For $\delta_{\Gamma}$ minimum degree of vertices of $\Gamma$ and maximum degree $\Delta_{\Gamma}$, using Lemma 2 and Proposition 2, we have

$$
\begin{gathered}
\sum_{\text {uv are pendentedges of } \Gamma} \sqrt{\frac{\operatorname{deg}_{u}+\operatorname{deg}_{v}-2}{\operatorname{deg}_{u} \operatorname{deg}_{v}}} \leq \frac{\sqrt{\Delta_{\Gamma}}}{\Delta_{\Gamma}+1} p, \\
\sum_{\substack{u v \in A \\
\operatorname{deg}_{u}+\operatorname{deg}_{u}+1 \\
\operatorname{deg}_{u} \text { or } \operatorname{deg}_{u} \geq 2}} \sqrt{\frac{\operatorname{deg}_{u}+\operatorname{deg}_{v}-2}{\operatorname{deg}_{u} \operatorname{deg}_{v}}} \leq k \Delta_{\Gamma} \frac{1}{\sqrt{2}},
\end{gathered}
$$




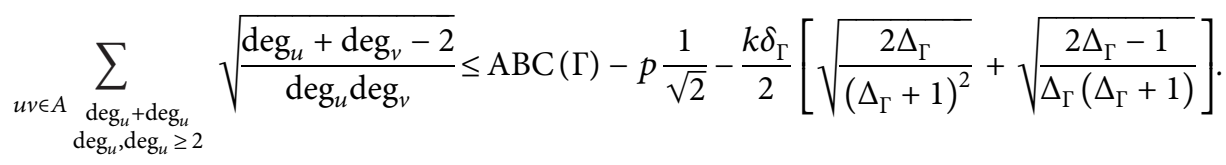

Now, from equation (35),

$$
\operatorname{ABC}\left(\Gamma_{n}^{k, l}\right) \leq \operatorname{ABC}(\Gamma)+p \sqrt{\frac{\Delta_{\Gamma}}{\left(\Delta_{\Gamma}+1\right)}}+k \Delta_{\Gamma} \sqrt{\frac{1}{2}}+\frac{\sqrt{2}}{2} k-\frac{k \delta_{\Gamma}}{2}\left[\sqrt{\frac{2 \Delta_{\Gamma}}{\left(\Delta_{\Gamma}+1\right)^{2}}}+\sqrt{\frac{2 \Delta_{\Gamma}-1}{\Delta_{\Gamma}\left(\Delta_{\Gamma}+1\right)}}\right]-\frac{\sqrt{2}}{2} p+\frac{\sqrt{2}}{2} k(l-2)+\frac{\sqrt{2}}{2} k .
$$

After simplification,

$$
\operatorname{ABC}\left(\Gamma_{n}^{k, l}\right) \leq k \Delta_{\Gamma} \sqrt{\frac{1}{2}}-\frac{k \delta_{\Gamma}}{2}\left[\sqrt{\frac{2 \Delta_{\Gamma}}{\left(\Delta_{\Gamma}+1\right)^{2}}}+\sqrt{\frac{2 \Delta_{\Gamma}-1}{\Delta_{\Gamma}\left(\Delta_{\Gamma}+1\right)}}\right]+\frac{\sqrt{2}}{2} \mathrm{kl}+p\left(\sqrt{\frac{\Delta_{\Gamma}}{\left(\Delta_{\Gamma}+1\right)}}-\frac{\sqrt{2}}{2}\right)+\mathrm{ABC}(\Gamma)
$$

Inequality (38) completes the proof.

Theorem 4 gives the discussion about the effect of successive applications of transformation $A$ as shown in Figure 2 over $\mathrm{ABC}$ index.
Theorem 4. Let graph $\Gamma_{n}^{k, l}$ with maximum degree of $\Delta_{\Gamma}+1$ and minimum $\delta_{\Gamma}$. Then,

$$
\begin{aligned}
\operatorname{ABC}\left(A_{\alpha}\left(\Gamma_{n}^{k, l}\right)\right) \leq & \frac{1}{\sqrt{2}} \mathrm{kl}+k \alpha \sqrt{\frac{\Delta_{\Gamma}+\alpha}{\left(\Delta_{\Gamma}+\alpha+1\right)}}+k \Delta_{\Gamma} \sqrt{\frac{2 \delta_{\Gamma}+2 \alpha-1}{\left(\delta_{\Gamma}+\alpha\right)\left(\delta_{\Gamma}+\alpha+1\right)}} \\
& -\frac{k \delta_{\Gamma}}{2}\left[\sqrt{\frac{2 \Delta_{\Gamma}+2 \alpha}{\left(\Delta_{\Gamma}+\alpha+1\right)^{2}}}+\sqrt{\frac{2 \Delta_{\Gamma}+2 \alpha-1}{\left(\Delta_{\Gamma}+\alpha\right)\left(\Delta_{\Gamma}+\alpha+1\right)}}\right] \\
& +p\left(\sqrt{\frac{\Delta_{\Gamma}+\alpha}{\left(\Delta_{\Gamma}+\alpha+1\right)}}-\frac{1}{\sqrt{2}}\right)+\operatorname{ABC}(\Gamma) .
\end{aligned}
$$

Equality holds for $\Gamma$ a complete graph of size $n$ with pendent paths of length $l$ at each vertex, i.e., $k=n$.
Proof. Let $\Gamma_{n}^{k, l}$ graph having pendent paths and $A_{\alpha}$ be the $\alpha$ time repetition of transformation $A . \operatorname{ABC}(\Gamma)$ is 


$$
\operatorname{ABC}(\Gamma)=\sum_{u v \in E(\Gamma)} \sqrt{\frac{\operatorname{deg}_{u}+\operatorname{deg}_{v}-2}{\operatorname{deg}_{u} \operatorname{deg}_{v}}} .
$$

After successive applications of transformation $A$ as $A_{\alpha}$, $\alpha \leq l-1$, the edge set of $A_{\alpha}\left(\Gamma_{n}^{k, l}\right)$ is partitioned as $E_{\left(\operatorname{deg}_{u}+\operatorname{deg}_{v}\right)}\left(A_{\alpha}\left(\Gamma_{n}^{k, l}\right)\right)$ where

$$
\left(\operatorname{deg}_{u}+\operatorname{deg}_{v}\right) \in\left\{3,4, \operatorname{deg}_{u}+\alpha+2, \operatorname{deg}_{u}+\alpha+3, \operatorname{deg}_{u}+\operatorname{deg}_{v}, \operatorname{deg}_{u}+\alpha+1+\operatorname{deg}_{v}\right\}
$$

The construction of $A_{\alpha}\left(\Gamma_{n}^{k, l}\right)$ shows

$$
\begin{aligned}
& E_{3}\left(A_{\alpha}\left(\Gamma_{n}^{k, l}\right)\right)=\left\{\mathrm{uv} \in \Gamma_{n}^{k, l}: \operatorname{deg}_{u}=1, \operatorname{deg}_{v}=2\right\}, \\
& E_{4}\left(A_{\alpha}\left(\Gamma_{n}^{k, l}\right)\right)=\left\{\mathrm{uv} \in \Gamma_{n}^{k, l}: \operatorname{deg}_{u}=\operatorname{deg}_{v}=2\right\}, \\
& E_{\operatorname{deg}_{u}+\alpha+2}\left(A_{\alpha}\left(\Gamma_{n}^{k, l}\right)\right)=\left\{\mathrm{uv} \in \Gamma_{n}^{k, l}: \delta_{\Gamma}+1 \leq \operatorname{deg}_{u}=\operatorname{deg}_{u}+\alpha+1 \leq \Delta_{\Gamma}+\alpha+1, \operatorname{deg}_{v}=1\right\} \text {, } \\
& E_{\operatorname{deg}_{u}+\alpha+3}\left(A_{\alpha}\left(\Gamma_{n}^{k, l}\right)\right)=\left\{\mathrm{uv} \in \Gamma_{n}^{k, l}: \delta_{\Gamma}+1 \leq \operatorname{deg}_{u}=\operatorname{deg}_{u}+\alpha+1 \leq \Delta_{\Gamma}+\alpha+1, \operatorname{deg}_{v}=2\right\} \\
& E_{\operatorname{deg}_{u}+\operatorname{deg}_{v}}\left(A_{\alpha}\left(\Gamma_{n}^{k, l}\right)\right)=\left\{\mathrm{uv} \in \Gamma_{n}^{k, l}: \delta_{\Gamma} \leq \operatorname{deg}_{u}=\operatorname{deg}_{u}, \operatorname{deg}_{v}=\operatorname{deg}_{v} \leq \Delta_{\Gamma}\right\}, \\
& E_{\operatorname{deg}_{u}+\alpha+1+\operatorname{deg}_{v}}\left(A_{\alpha}\left(\Gamma_{n}^{k, l}\right)\right)=\left\{\mathrm{uv} \in \Gamma_{n}^{k, l}: \delta_{\Gamma} \leq \operatorname{deg}_{v}=\operatorname{deg}_{v}, \operatorname{deg}_{u} \leq \Delta_{\Gamma}, \operatorname{deg}_{u}=\operatorname{deg}_{u}+\alpha+1\right\} \text {, } \\
& \operatorname{ABC}\left(A_{\alpha}\left(\Gamma_{n}^{k, l}\right)\right)=\sum_{\text {uv are edges of pendent paths }} \sqrt{\frac{\operatorname{deg}_{u}+\operatorname{deg}_{v}-2}{\operatorname{deg}_{u} \operatorname{deg}_{v}}}+\sum_{\text {uv are edges of } \Gamma} \sqrt{\frac{\operatorname{deg}_{u}+\operatorname{deg}_{v}-2}{\operatorname{deg}_{u} \operatorname{deg}_{v}}}, \\
& \operatorname{ABC}\left(A_{\alpha}\left(\Gamma_{n}^{k, l}\right)\right)=\sum_{u v \in A_{3}} \sqrt{\frac{\operatorname{deg}_{u}+\operatorname{deg}_{v}-2}{\operatorname{deg}_{u} \operatorname{deg}_{v}}}+\sum_{u v \in A_{4}} \sqrt{\frac{\operatorname{deg}_{u}+\operatorname{deg}_{v}-2}{\operatorname{deg}_{u} \operatorname{deg}_{v}}}+\sum_{u v \in A_{\text {deg }_{u}+\alpha+2}} \sqrt{\frac{\operatorname{deg}_{u}+\operatorname{deg}_{v}-2}{\operatorname{deg}_{u} \operatorname{deg}_{v}}}+\sum_{u v \in A_{\operatorname{deg}_{u}+\alpha+3}} \sqrt{\frac{\operatorname{deg}_{u}+\operatorname{deg}_{v}-2}{\operatorname{deg}_{u} \operatorname{deg}_{v}}} \\
& +\sum_{\text {uv are pendentedges of } \Gamma} \sqrt{\frac{\operatorname{deg}_{u}+\operatorname{deg}_{v}-2}{\operatorname{deg}_{u} \operatorname{deg}_{v}}}+\sum_{u v \in A_{\operatorname{deg}_{u}+\operatorname{deg}_{v}}} \sqrt{\frac{\operatorname{deg}_{u}+\operatorname{deg}_{v}-2}{\operatorname{deg}_{u} \operatorname{deg}_{v}}}+\sum_{u v \in A_{\operatorname{deg}_{u}+\operatorname{deg}_{u}+\alpha+1}} \sqrt{\frac{\operatorname{deg}_{u}+\operatorname{deg}_{v}-2}{\operatorname{deg}_{u}+\operatorname{deg}_{u} \geq 2}} \\
& \left|E_{3}\left(A_{\alpha}\left(\Gamma_{n}^{k, l}\right)\right)\right|=k \\
& \left|E_{4}\left(A_{\alpha}\left(\Gamma_{n}^{k, l}\right)\right)\right|=k(l-\alpha-2), \\
& \left|E_{\operatorname{deg}_{u}+\alpha+2}\left(A_{\alpha}\left(\Gamma_{n}^{k, l}\right)\right)\right|=k \alpha \\
& \begin{array}{l}
\left|E_{\operatorname{deg}_{u}+\alpha+3}\left(A_{\alpha}\left(\Gamma_{n}^{k, l}\right)\right)\right|=k, \\
\left|\begin{array}{c}
A \operatorname{deg}_{u}+\operatorname{deg}_{u}+1 \\
\operatorname{deg}_{u}, \operatorname{deg}_{u} \geq 2
\end{array}\right| \leq k \Delta_{\Gamma}-p,
\end{array}
\end{aligned}
$$


and $\left|A_{1+\operatorname{deg}_{u}}\right|=p$. For $\delta_{\Gamma}$ minimum degree of vertices of $\Gamma$ and maximum degree $\Delta_{\Gamma}$, using Lemma 2 and Proposition 2, we have

$$
\begin{aligned}
& \sum_{u v \text { are pendent edges of } \Gamma} \sqrt{\frac{\operatorname{deg}_{u}+\operatorname{deg}_{v}-2}{\operatorname{deg}_{u} \operatorname{deg}_{v}}} \leq p \sqrt{\frac{\Delta_{\Gamma}+\alpha}{\left(\Delta_{\Gamma}+\alpha+1\right)}}
\end{aligned}
$$

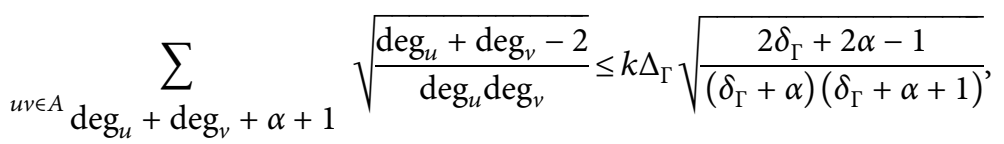

$$
\begin{aligned}
& \operatorname{deg}_{u}, \operatorname{deg}_{u} \geq 2 \\
& \sum_{u v \in A_{\operatorname{deg}_{u}+\alpha+2}} \sqrt{\frac{\operatorname{deg}_{u}+\operatorname{deg}_{v}-2}{\operatorname{deg}_{u} \operatorname{deg}_{v}}} \leq k \alpha \sqrt{\frac{\Delta_{\Gamma}+\alpha}{\left(\Delta_{\Gamma}+\alpha+1\right)}} \\
& \sum_{u v \in A_{\operatorname{deg}_{u}+\alpha+3}} \sqrt{\frac{\operatorname{deg}_{u}+\operatorname{deg}_{v}-2}{\operatorname{deg}_{u} \operatorname{deg}_{v}}}=\frac{1}{\sqrt{2}} k, \\
& \sum_{u v \in A} \operatorname{deg}_{u}+\operatorname{deg}_{u} \sqrt{\frac{\operatorname{leg}_{u}+\operatorname{deg}_{v}-2}{\operatorname{deg}_{u} \operatorname{deg}_{v}}} \leq \operatorname{ABC}(\Gamma)-p \frac{1}{\sqrt{2}}-\frac{k \delta_{\Gamma}}{2} \sqrt{\frac{2 \Delta_{\Gamma}+2 \alpha}{\left(\Delta_{\Gamma}+\alpha+1\right)^{2}}}-\frac{k \delta_{\Gamma}}{2} \sqrt{\frac{2 \Delta_{\Gamma}+2 \alpha-1}{\left(\Delta_{\Gamma}+\alpha\right)\left(\Delta_{\Gamma}+\alpha+1\right)}} \\
& \operatorname{deg}_{u}, \operatorname{deg}_{u} \geq 2
\end{aligned}
$$

Substituting these changes in equation (42), we have following inequality.

$$
\begin{aligned}
\operatorname{ABC}\left(A_{\alpha}\left(\Gamma_{n}^{k, l}\right)\right) \leq & p \sqrt{\frac{\Delta_{\Gamma}+\alpha}{\left(\Delta_{\Gamma}+\alpha+1\right)}}+\frac{1}{\sqrt{2}} k(l-2-\alpha)+\frac{1}{\sqrt{2}} k+k \alpha \sqrt{\frac{\Delta_{\Gamma}+\alpha}{\left(\Delta_{\Gamma}+\alpha+1\right)}}+k \Delta_{\Gamma} \sqrt{\frac{2 \delta_{\Gamma}+2 \alpha-1}{\left(\delta_{\Gamma}+\alpha\right)\left(\delta_{\Gamma}+\alpha+1\right)}+\mathrm{ABC}(\Gamma)} \\
& -p \frac{1}{\sqrt{2}}-\frac{k \delta_{\Gamma}}{2}\left[\sqrt{\frac{2 \Delta_{\Gamma}+2 \alpha}{\left(\Delta_{\Gamma}+\alpha+1\right)^{2}}}+\sqrt{\frac{2 \Delta_{\Gamma}+2 \alpha-1}{\left(\Delta_{\Gamma}+\alpha\right)\left(\Delta_{\Gamma}+\alpha+1\right)}}\right] .
\end{aligned}
$$

After simplification, we get

$$
\begin{aligned}
\operatorname{ABC}\left(A_{\alpha}\left(\Gamma_{n}^{k, l}\right)\right) \leq & \frac{1}{\sqrt{2}} \mathrm{kl}+k \alpha \sqrt{\frac{\Delta_{\Gamma}+\alpha}{\left(\Delta_{\Gamma}+\alpha+1\right)}}+k \Delta_{\Gamma} \sqrt{\frac{2 \delta_{\Gamma}+2 \alpha-1}{\left(\delta_{\Gamma}+\alpha\right)\left(\delta_{\Gamma}+\alpha+1\right)}}-\frac{k \delta_{\Gamma}}{2}\left[\sqrt{\left.\frac{2 \Delta_{\Gamma}+2 \alpha}{\left(\Delta_{\Gamma}+\alpha+1\right)^{2}}+\sqrt{\frac{2 \Delta_{\Gamma}+2 \alpha-1}{\left(\Delta_{\Gamma}+\alpha\right)\left(\Delta_{\Gamma}+\alpha+1\right)}}\right]}\right. \\
& +p\left(\sqrt{\frac{\Delta_{\Gamma}+\alpha}{\left(\Delta_{\Gamma}+\alpha+1\right)}}-\frac{1}{\sqrt{2}}\right)+\operatorname{ABC}(\Gamma) .
\end{aligned}
$$


Inequality (46) completes the proof.

\section{Conclusion}

The study of mathematical aspect regarding topological indices is a partially open problem: for which members of graph family, certain index has minimal or maximal value? In this work, we deal with this fundamental question. We considered graphs of family $\Gamma_{n}^{k, l}$ with $0 \leq k \leq n$ pendent paths of length $l \geq 2$ and transformed family $A_{\alpha}\left(\Gamma_{n}^{k, l}\right)$ where transformation $A_{\alpha}$ is the graph transformation. The concluding key points concerning our study are given as follows. We studied the fact of pendent paths over the increase and decrease of AZI and ABC index in addition to defining upper bounds for these indices and mentioned graphs with maximum values of these indices. We discussed the fact of defined transformation as $A_{\alpha} ; 0 \leq \alpha \leq l-2,0 \leq k \leq n$ over $\mathrm{AZI}$ and $\mathrm{ABC}$ indices. We determined upper bounds and characterized extremal graphs for such bounds.

\section{Data Availability}

No data were used to support this study.

\section{Conflicts of Interest}

The authors declare that they have no conflicts of interest.

\section{Authors' Contributions}

All authors contributed equally to this study.

\section{Acknowledgments}

The authors deeply acknowledge the Researchers Supporting Program (TUMA-Project-2021-27) Almaarefa University, Riyadh, Saudi Arabia, for supporting steps of this work.

\section{References}

[1] J. B. Liu, H. Shaker, I. Nadeem, and M. Hussain, "Topological aspects of boron nanotubes," Advances in Materials Science and Engineering, vol. 2018, 2018.

[2] S. Mondal, A. Dey, N. De, and A. Pal, "QSPR analysis of some novel neighbourhood degree-based topological descriptors," Complex \& Intelligent Systems, vol. 7, no. 2, pp. 977-996, 2021.

[3] G. Rücker and C. Rücker, "On topological indices, boiling points, and cycloalkanes," Journal of Chemical Information and Computer Sciences, vol. 39, no. 5, pp. 788-802, 1999.

[4] H. Wiener, "Structural determination of paraffin boiling points," Journal of the American Chemical Society, vol. 69, no. 1, pp. 17-20, 1947.

[5] M. Randic, "Characterization of molecular branching," Journal of the American Chemical Society, vol. 97, no. 23, pp. 6609-6615, 1975.

[6] I. Gutman and N. Trinajstić, "Graph theory and molecular orbitals. total $\varphi$-electron energy of alternant hydrocarbons," Chemical Physics Letters, vol. 17, no. 4, pp. 535-538, 1972.

[7] B. Bollobás and P. Erdos, "Graphs of extremal weights," Ars Combinatoria, vol. 50, pp. 225-233, 1998.
[8] E. Estrada, L. Torres, L. Rodriguez, and I. Gutman, “An atombond connectivity index: modelling the enthalpy of formation of alkanes," Indian Journal of Chemistry, vol. 37A, 1998.

[9] D. Vukičević and B. Furtula, "Topological index based on the ratios of geometrical and arithmetical means of end-vertex degrees of edges," Journal of Mathematical Chemistry, vol. 46, no. 4, pp. 1369-1376, 2009.

[10] Y. Zheng, W. Lin, W. Lin, Q. A. Chen, L. Huang, and Z. Wu, "Characterizing trees with minimal $\mathrm{ABC}$ index with computer search: a short survey," Open Journal of Discrete Applied Mathematics, vol. 1, no. 2, pp. 1-9, 2018.

[11] K. C. Das, S. Elumalai, and I. Gutman, "On ABC index of graphs. match Commun,” Math. Comput. Chem, vol. 78, pp. 459-468, 2017.

[12] B. Furtula, A. Graovac, and D. Vukičević, "Augmented zagreb index," Journal of Mathematical Chemistry, vol. 48, no. 2, pp. 370-380, 2010.

[13] I. Gutman and J. Tosovic, "Testing the quality of molecular structure descriptors. vertex-degree-based topological indices," Journal of the Serbian Chemical Society, vol. 78, no. 6, pp. 805-810, 2013.

[14] I. Gutman, "Degree-based topological indices," Croatica Chemica Acta, vol. 86, no. 4, pp. 351-361, 2013.

[15] Y. Huang, B. Liu, and L. Gan, "Augmented zagreb index of connected graphs," Match-Communications in Mathematical and Computer Chemistry, vol. 67, no. 2, 2012.

[16] M. B. Ahmadi, D. Dimitrov, I. Gutman, and S. A. Hosseini, "Disproving a conjecture on trees with minimal atom-bond connectivity index," Match Commun. Math. Comput. Chem, vol. 72, no. 3, pp. 685-698, 2014.

[17] M. Bianchi, A. Cornaro, J. L. Palacios, and A. Torriero, "New upper bounds for the ABC index," Match Commun. Math. Comput. Chem, vol. 76, no. 1, pp. 117-130, 2016.

[18] K. C. Das, I. Gutman, and B. Furtula, "On atom-bond connectivity index," Chemical Physics Letters, vol. 511, no. 4-6, pp. 452-454, 2011.

[19] K. C. Das, "Maximizing the sum of the squares of the degrees of a graph," Discrete Mathematics, vol. 285, no. 1-3, pp. 57-66, 2004.

[20] K. C. Das, “Atom-bond connectivity index of graphs," Discrete Applied Mathematics, vol. 158, no. 11, pp. 1181-1188, 2010.

[21] D. Dimitrov, "Efficient computation of trees with minimal atom-bond connectivity index," Applied Mathematics and Computation, vol. 224, pp. 663-670, 2013.

[22] D. Dimitrov, "On structural properties of trees with minimal atom-bond connectivity index," Discrete Applied Mathematics, vol. 172, pp. 28-44, 2014.

[23] D. Dimitrov, Z. Du, and C. M. D. Fonseca, "On structural properties of trees with minimal atom-bond connectivity index III: Trees with pendent paths of length three," Applied Mathematics and Computation, vol. 282, pp. 276-290, 2016.

[24] I. Gutman and B. Furtula, "Trees with smalleuvatom-bond connectivity index," Match-Communications in Mathematical and Computer Chemistry, vol. 68, no. 1, 2012.

[25] I. Gutman, J. Tošović, S. Radenković, and S. Marković, "On atom-bond connectivity index and its chemical applicability," Indian Journal of Chemistry, vol. 51A, 2012.

[26] D. Wang, Y. Huang, and B. Liu, "Bounds on augmented Zagreb index," MATCH Commun. Math. Comput. Chem, vol. 68, pp. 209-216, 2012.

[27] Y. Huang, B. Liu, and L. Gan, "Augmented zagreb index of connected graphs," Match Commun. Math. Comput. Chem.vol. 67, pp. 483-494, 2012. 
[28] F. Zhan, Y. Qiao, and J. Cai, "Unicyclic and bicyclic graphs with minimal augmented zagreb index," Journal of Inequalities and Applications, vol. 2015, no. 1, 2015.

[29] Y. Huang and B. Liu, "Ordering graphs by the augmented zagreb indices,” J. Math. Res. Appl.vol. 2, pp. 119-129, 2015.

[30] F. Zhan, W. Wang, J. Cai, and Y. Qiao, "The augmented zagreb index of catacondensed systems," J. Beijing Normal Univ. (Natural Sci.), vol. 4, pp. 340-347, 2015.

[31] A. Ali, A. A. Bhatti, and Z. Raza, "The augmented zagreb index, vertex connectivity and matching number of graphs," Bull. Iran. Math. Soc.vol. 42, pp. 417-425, 2016.

[32] A. Ali and A. A. Bhatti, "A note on the augmented zagreb index of cacti with fixed number of vertices and cycles," Kuwait J. Sci.vol. 43, pp. 11-17, 2016.

[33] J. L. Palacios, "Bounds for the augmented zagreb and the atom-bond connectivity indices," Applied Mathematics and Computation, vol. 307, pp. 141-145, 2017.

[34] A. Ali, Z. Raza, and A. A. Bhatti, "On the augmented zagreb index," Kuwait J. Sci.vol. 43, pp. 48-63, 2016.

[35] X. Sun, Y. Gao, J. Du, and L. Xu, "Augmented zagreb index of trees and unicyclic graphs with perfect matchings," Applied Mathematics and Computation, vol. 335, pp. 75-81, 2018.

[36] C. K. Gupta, V. Lokesha, S. B. Shwetha, and P. S. Ranjini, "On the symmetric division deg index of graph," Southeast Asian Bulletin of Mathematics, vol. 40, no. 1, 2016.

[37] M. Asif, M. Hussain, H. Almohamedh, K. M. Alhamed, and S. Almotairi, "An approach to the extremal inverse degree index for families of graphs with transformation effect," Journal of Chemistry, vol. 2021, Article ID 6657039, 8 pages, 2021.

[38] M. Asif, H. Almohamedh, M. Hussain, K. M. Alhamed, A. A. Almutairi, and S. Almotairi, "An approach to the geometric-arithmetic index for graphs under transformations' fact over pendent paths," Complexity, vol. 2021, Article ID 3745862, 13 pages, 2021.

[39] Z. Shao, P. Wu, Y. Gao, I. Gutman, and X. Zhang, "On the maximum ABC index of graphs without pendent vertices," Applied Mathematics and Computation, vol. 315, pp. 298-312, 2017.

[40] R. Xing, B. Zhou, and Z. Du, "Further results on atom-bond connectivity index of trees," Discrete Applied Mathematics, vol. 158, no. 14, pp. 1536-1545, 2010. 